\title{
Visual Selection and the Human Frontal Eye Fields: Effects of Frontal Transcranial Magnetic Stimulation on Partial Report Analyzed by Bundesen's Theory of Visual Attention
}

\author{
June Hung, ${ }^{1,2}$ Jon Driver, ${ }^{2}$ and Vincent Walsh ${ }^{2}$ \\ ${ }^{1}$ Department of Neurology, Chang Gung Memorial Hospital and Chang Gung University College of Medicine, Taipei 10507, Taiwan, and ${ }^{2}$ Institute of \\ Cognitive Neuroscience, University College London, London WC1N 3AR, United Kingdom
}

While the frontal eye fields (FEF) are traditionally associated with eye movements, recent work indicates possible roles in controlling selective visual processing. We applied $10 \mathrm{~Hz}$ bursts of transcranial magnetic stimulation (TMS) over left or right human FEF while subjects performed a partial-report task that allowed quantitative estimates of top-down control and other parameters affecting visual performance. Participants selectively reported digits in a relevant color (targets) but not those in an irrelevant color (nontargets) from a brief masked display. A target could appear alone or together with an accompanying item (nontarget or target) in the same or opposite hemifield. Targets were normally identified better when presented with a nontarget than with another target, indicating prioritization of task-relevant targets and thus top-down control. We found this usual pattern of results without TMS, and also with TMS over left FEF. However, during right FEF TMS, the detrimental impact of accompanying distractors increased. Formal analysis in terms of Bundesen's (1990) theory of visual attention confirmed that right FEF TMS diminished the top-down control parameter for both hemifields, indicating an FEF role in top-down selection even for targets defined by the nonspatial property of color. Direct comparison with our previous findings for parietal TMS (Hung et al., 2005) confirmed the distinct role of FEF in top-down control, plus right-hemisphere predominance for this in humans.

\section{Introduction}

Functions of the frontal eye fields (FEF) have been reassessed in recent years. Although classically described as important for eye movements, FEF also play important roles in visual processing. Early anatomical models of visual cortex (Maunsell and Van Essen, 1987) placed the FEF at the pinnacle of the visual system, but recent connectivity analyses (Barone et al., 2000) show intimate connections of FEF with extrastriate occipital cortices. Moreover, single-cell studies now show involvement of FEF in visual detection and priming (Schall and Hanes, 1993; Thompson et al., 1996, 1997; Schall and Bichot, 1998; Bichot and Schall, 1999, 2002; Schall and Thompson, 1999; Thompson and Schall, 1999; Murthy et al., 2001). Microstimulation of monkey FEF during single-unit recordings from V4 has revealed influences of FEF on occipital activity (Moore and Fallah, 2001; Moore and Armstrong, 2003).

\footnotetext{
Received May 26, 2011; revised July 29, 2011; accepted Aug. 23, 2011.

Author contributions: J.H., J.D., and V.W. designed research; J.H. performed research; J.H., J.D., and V.W. contributed unpublished reagents/analytic tools; J.H. analyzed data; J.H., J.D., and V.W. wrote the paper.

This work was supported by the Wellcome Trust and the Medical Research Council (V.W. and J.D.), the Royal Society (V.W. and J.D.), the Swire Education Trust (J.H.), National Science Council of Taiwan (NSC-96-2413-H-182A001-MY2; to J.H.), and. Chang Gung Memorial Hospital (CMRPG 350933; to J.H.). We thank C. Bundesen and S. Kyllingsbæk for providing the program computing TVA parameters.

The authors declare no financial conflicts of interest.

Correspondence should be addressed to Vincent Walsh, Institute of Cognitive Neuroscience, University College London, 17 Queen Square, London WC1N 3AR, UK. E-mail: v.walsh@ucl.ac.uk.

DOI:10.1523/JNEUROSCI.2626-11.2011

Copyright $\odot 2011$ the authors $\quad 0270-6474 / 11 / 3115904-10 \$ 15.00 / 0$
}

These monkeys studies have been corroborated and extended by human studies applying transcranial magnetic stimulation (TMS) to human FEF, suggesting roles for FEF in visual orienting, search, and priming (Muggleton et al., 2003; O'Shea et al., 2004; Smith et al., 2005; Campana et al., 2007). Moreover, TMS over human FEF can change visual-evoked potentials recorded at occipital electrodes (Taylor et al., 2007) and affect activity in retinotopic human visual cortex as assessed with concurrent functional magnetic resonance imaging (fMRI) during TMS (Ruff et al., 2006). While human TMS studies converge with monkey neurophysiology in demonstrating roles for FEF in modulating visual processing that go beyond purely oculomotor contributions, one distinctive aspect of the human studies is that TMS applied over right human FEF is typically much more effective in affecting visual processing than TMS over left FEF (the above TMS studies; Grosbras and Paus, 2003; Silvanto et al., 2006). This may reflect some right-hemisphere predominance in networks modulating visual processing, which might be specific to humans (Ruff et al., 2009), as also suggested by an extensive clinical literature showing that right-hemisphere lesions to frontal (and/or parietal) cortex often disrupt visual function more than comparable left lesions in humans (Mesulam, 1999; Vuilleumier and Driver, 2007).

Although there is an emerging consensus that FEF (particularly in the right hemisphere, for humans) can modulate visual processing, the exact nature of this FEF contribution needs better characterization in formal psychological and computational terms. Here we examined effects of TMS bursts over left or right 
A
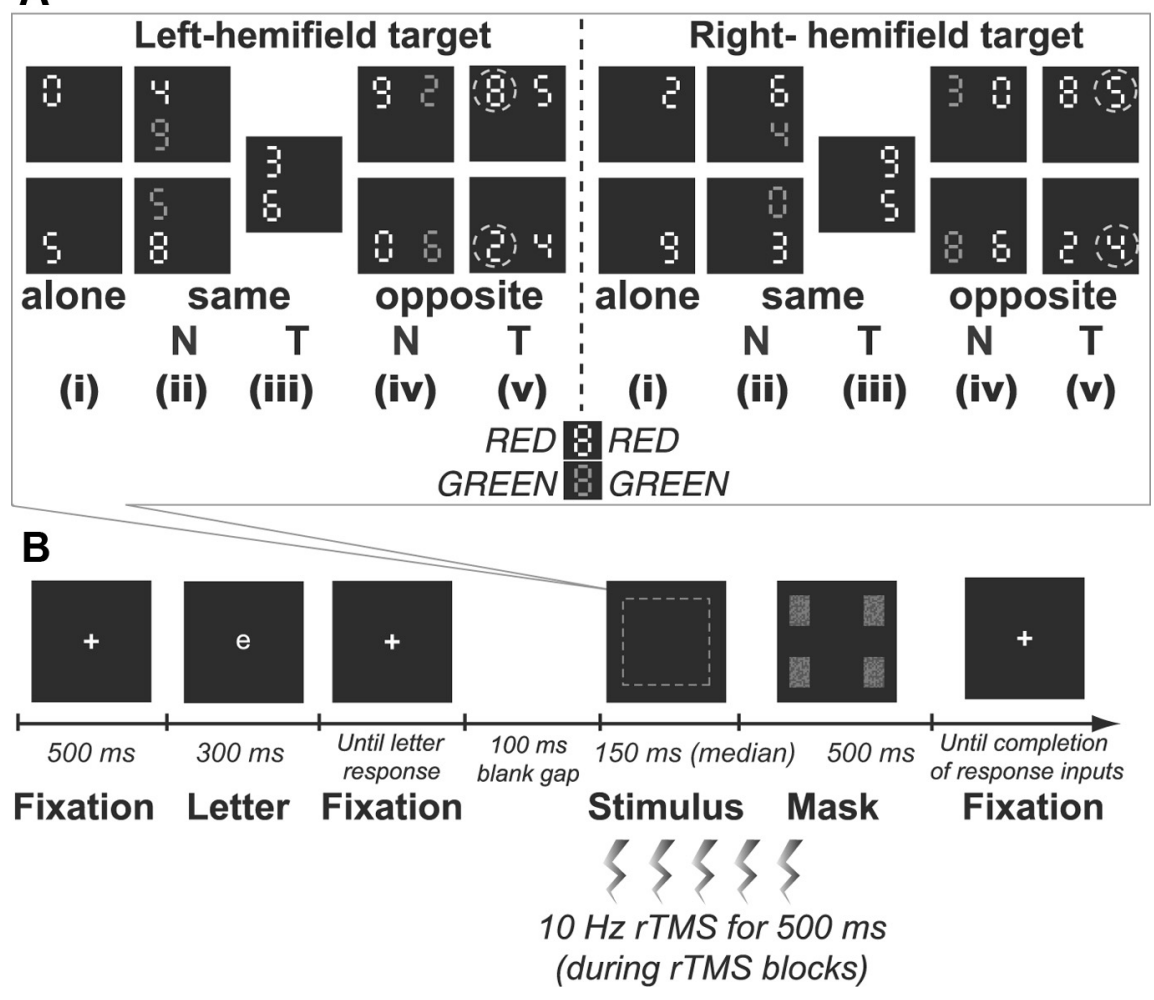

Figure 1. A, Example stimulus displays for the selective partial-report task. Participants reported digit identities in the taskrelevant color (red or green, depending on the blocked instruction; in the illustrated examples, red items are targets). Each black-background square shown corresponds to one possible display type. Targets could appear alone (i), accompanied by a same-hemifield nontarget (ii) or target (iii), in bilateral displays with a nontarget (iv), or with an additional target in the other hemifield (v). Dashed circles indicate which target item was scored for a given display condition (they did not appear during the task). N, nontarget; T, target. $\boldsymbol{B}$, Temporal sequence of an example single trial. Participants first reported a small white letter at fixation to encourage appropriate fixation (monitored by eye-tracking). Next was a brief stimulus display (dashed square, which corresponds to one of the different display types illustrated in $A$ ), then a mask. Any TMS was given as five successive bursts at $10 \mathrm{~Hz}$ beginning at onset of the main stimulus display. Responses were given verbally and recorded by the experimenter. All these aspects were equivalent to Hung et al. (2005).

human FEF upon performance in a selective partial-report task suitable for formal analysis via Bundesen's (1990) mathematical theory of visual attention (TVA). This allowed separate parameters concerning top-down control, sensory effectiveness, and attentional weight to be measured within a single visual task (see Materials and Methods, Data analysis, below). We have previously examined the impact of parietal TMS on Bundesen parameters in this task (Hung et al., 2005). Applying TMS to the left or right human FEF permitted a new comparison between the frontal effects studied here and the parietal effects reported previously (Hung et al., 2005) to further specify the distinct roles of different nodes in the network modulating visual processing.

\section{Materials and Methods}

Participants. Eight healthy volunteers (age 26-35 years, three female) gave informed consent. All were right-handers (Oldfield, 1971) and selfreported normal or corrected-to-normal vision. The protocol was approved by the local ethics committee and conformed to the Declaration of Helsinki and safety guidelines for repetitive TMS research (Wassermann, 1998; Rossi et al., 2009).

Stimuli and procedures. The general procedures were modified from a previous study (Duncan et al., 1999) that applied Bundesen's (1990) TVA approach to study effects of permanent brain damage in patients. The exact procedures adhered to those used in our previous parietal TMS study of healthy volunteers (Hung et al., 2005) for the selective partialreport paradigm, being identical to that study in all respects except for the new TMS sites used here [now over left or right $\mathrm{FEF}$, rather than over left or right posterior parietal cortex (PPC)]. Our use of equivalent methods, including identical TMS parameters except for the changed TMS sites, allowed a direct comparison of the present new FEF effects to the PPC effects found previously. Given the similarity in methods, we summarize the protocol only briefly here; see Hung et al. (2005) for further details.

The stimulus array was comprised of one or two digits $\left(\sim 0.5^{\circ}\right.$ in height $)$ at the corners of an imaginary square $\left(9.4^{\circ} \times 9.4^{\circ}\right)$ centered on fixation against a black background (Fig. 1A). Digits were randomly chosen from 02345689 and presented in red [Commission Internationale de l' Eclairage (CIE), $x=0.618, y=$ 0.346 ] and/or green (CIE, $x=0.279, y=0.577$ ) of comparable luminance $\left(20 \mathrm{~cd} / \mathrm{m}^{2}\right)$. At the start of each session, participants were instructed that either the red or green digits would serve as targets. The target was thus flexibly defined by color in a session-blocked instruction-dependent manner. Figure $1 \mathrm{~A}$ illustrates the possible conditions of presentation. A target item could appear alone (four display types, corresponding to each corner of the imaginary square); accompanied by a nontarget (eight display types, with a target at four possible positions and the nontarget appearing in the same or opposite hemifield), or accompanied by another target (four display types, corresponding to same-hemifield target columns on either side or opposite-hemifield rows above or below). Diagonal arrangements were not possible in our two-item displays (Fig. $1 A$ ). Each type of display was presented equally often in a random order.

As illustrated in Figure $1 B$, each trial began with a central white cross $\left(\sim 0.3^{\circ}\right)$. To further encourage central fixation, this was replaced for $300 \mathrm{~ms}$ by a small central white letter $\left(\sim 0.5^{\circ}\right.$ in height) that participants read aloud. The experimenter then pressed a key and $100 \mathrm{~ms}$ later the stimulus array was presented briefly (median duration $150 \mathrm{~ms}$; see below). This array was masked by a rectangular pattern with random pixels in red and green. Participants verbally reported the identity of any digits in the specified target color (i.e., reporting red digits while ignoring any green digits, or vice-versa, depending on current task instructions). For displays presenting two target digits, participants had to report both in any order and then indicate their order of report (e.g., which was on left, which on right, for bilateral target displays). Their responses were keyed in by the experimenter. All these aspects were as in Hung et al. (2005).

Head position was fixed by a chinrest and head strap. Stimuli appeared on a 17 inch VGA display (Sony Trinitron Multiscan 200 GS) at $100 \mathrm{~Hz}$ and $1024 \times 768$ pixel resolution, at a viewing distance of $57 \mathrm{~cm}$, under the control of E-prime 1.0 software (Psychology Software Tools). Eye position was monitored by an infrared limbus tracker (IRIS; Skalar Medical) and analyzed with DASYLab 5.0 (DATALOG). This confirmed that all participants maintained central fixation adequately $\left(<2^{\circ}\right.$ deviation within each brief display, no differences between conditions) and that TMS did not trigger blinking responses.

Over four separate days, participants performed four sessions of this task, two with red as the target color and two with green as the target color. Each session lasted for $\sim 2-3 \mathrm{~h}$ and consisted of an initial practice phase (presenting only four types of target-alone displays) followed by four experimental blocks (presenting all 16 types of displays). During the practice phase, a staircase procedure (Cornsweet, 1962) derived the exposure duration of the digit display that led to $75 \%$ accuracy over 16 


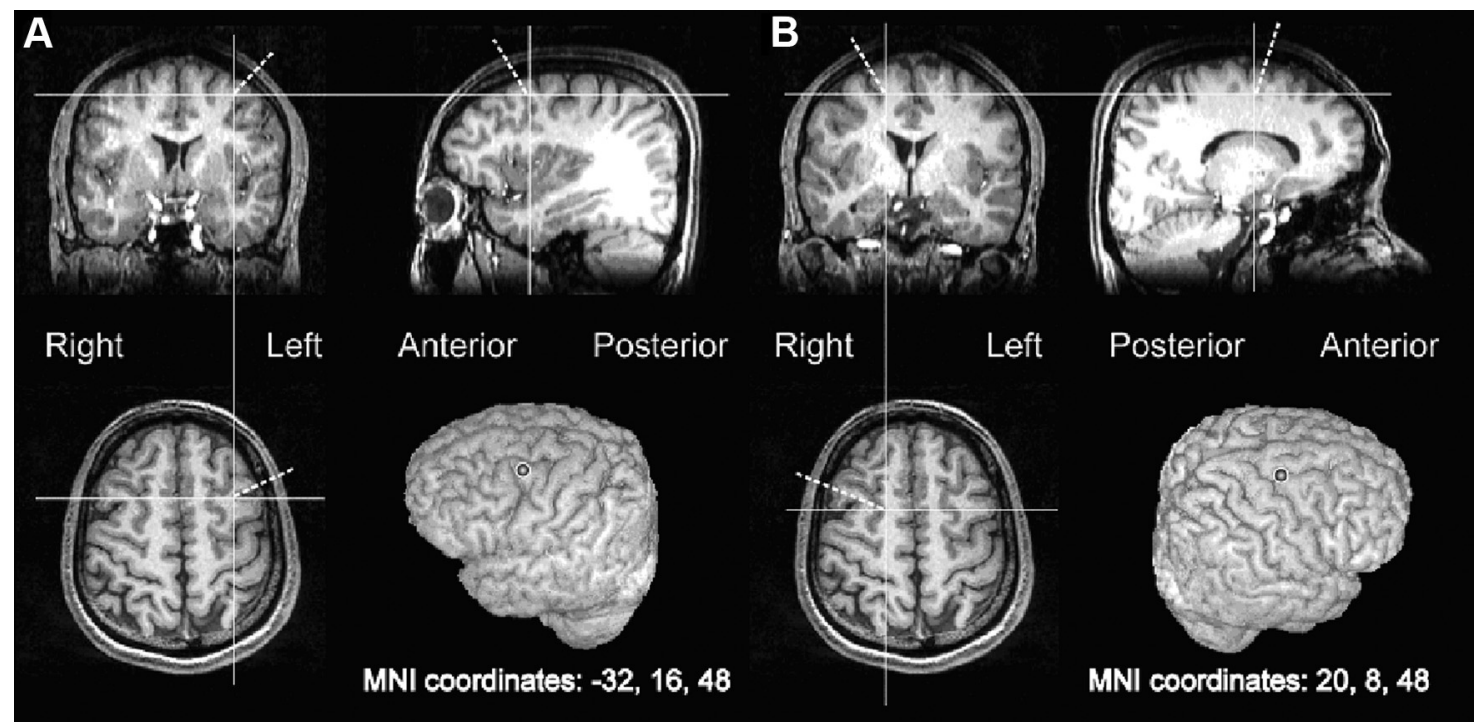

Figure 2. The targeted location over the frontal eye field in the left $(\boldsymbol{A})$ or right $(\boldsymbol{B})$ hemisphere of an illustrative participant, as derived from transforming the mean $\mathrm{MNI}$ coordinates in the middle frontal gyrus (Hopfinger et al., 2000) to the structural image of the participant (see Materials and Methods).

consecutive target-alone trials. At the end of every eight-trial cycle (over which four types of target-alone displays were presented equally often), the program calculated the averaged accuracy. If this accuracy was more or less than $75 \%$, the duration of the stimulus array was reduced or increased respectively by $10 \mathrm{~ms}$ for the next cycle of eight trials; if the accuracy was equal to $75 \%$, the same duration was maintained for the next eight trials. The staircase ended when a given exposure duration produced the same $75 \%$ accuracy over 16 consecutive trials, i.e., over two cycles. If the staircase terminated rather quickly (within 32 trials/4 cycles), it was rerun to ensure that the obtained duration was appropriate.

The chosen display duration was then maintained for four experimental blocks: two with no TMS, one with left FEF TMS, and one with right FEF TMS. These experimental blocks were arranged in pseudorandom order with $10 \mathrm{~min}$ breaks between them. Given that each single session used the same display duration for all TMS conditions (no TMS, right or left FEF TMS) and that the durations were functionally comparable (i.e., all set at $75 \%$ accuracy for target-alone trials without TMS), data collected across sessions were pooled together. Each block consisted of 128 trials, yielding a total of 1024 trials for the no TMS condition and 512 trials for right or left FEF TMS conditions per participant.

Color-report control task. To ensure that any TMS effects on selection by task-relevant color in the above partial-report task could not be due to any effects on gross color perception, we also tested a control task requiring report of stimulus color rather than digit identity (Duncan et al., 1999; Habekost and Bundesen, 2003; Hung et al., 2005). The stimulus array now comprised a single digit in red or green at one of the four possible positions (hence eight display types); these positions were identical to those used in the partial-report task. Each type of display was equally probable. For this task, participants gave verbal reports for the color of each single digit presented (i.e., red or green) in one session, which began with a practice phase that applied the usual staircase procedure to determine the exposure duration equivalent to $75 \%$ accuracy, followed by four experimental blocks in pseudorandom order. The four blocks consisted of two blocks without and two blocks with FEF TMS (one for the left and one for the right FEF), with 64 trials in each block.

Localization of the TMS sites. The coordinates for applying frontal TMS over human FEF were initially taken from an fMRI study (Hopfinger et al., 2000) that used a cueing paradigm for functional localization of the putative human FEF regions involved in selective attention. This putative human FEF region, falling in the posterior middle frontal gyrus, accord reasonably well with monkey studies and other anatomical work (for meta-analysis review of FEF location, see Paus, 1996; Tehovnik et al., 2000). The actual left and right coordinates (Fig. 2) fall within the typical human FEF regions, as defined by various human studies, including low-intensity electrical stimulation (Blanke et al., 2000) or neuroimaging work (Kawashima et al., 1998; Ruff et al., 2006). The coordinates used for our TMS sites here also accord well with those used in many other recent TMS studies of human FEF (Muggleton et al., 2003; O'Shea et al., 2004; Taylor et al., 2007; Ruff et al., 2009).

Protocol for TMS. We derived left and right TMS sites for each of our individual subjects at the same (normalized) Montreal Neurological Institute (MNI) coordinates ( $x, y, z=-32,16,48$ and 20, 8, 48; Fig. 2). Each participant's $\mathrm{T}_{1}$-weighted structural image was first spatially normalized to Talairach space (Talairach and Tournoux, 1988) with FSL software (www.fmrib.ox.ac.uk/fsl; Jenkinson and Smith, 2001) that uses the canonical MNI template (average of $152 \mathrm{~T}_{1}$-weighted stereotaxic volumes). We then applied an inverse transformation matrix, which accepts the chosen MNI coordinates as the input and gives the corresponding coordinates in the participant's native structural image as the output. These output coordinates were marked on the structural image in Brainsight software (Rogue Research), which, with the aid of a Polaris infrared tracking device (Northern Digital), can view the position of the TMS coil relative to the structure of each individual's head and brain.

Magnetic stimulation was generated by a Super Rapid stimulator (Magstim) connected to a $50 \mathrm{~mm}$ figure-of-eight coil held by a mechanical arm. For blocks with TMS, magnetic stimulation was delivered synchronously with the onset of the stimulus array to be reported (during the partial-report or the color-report control task) in a five-burst train at $10 \mathrm{~Hz}$ for $500 \mathrm{~ms}$, with a fixed intensity of $60 \%$ of maximum stimulator output (corresponding to $86-115 \%$ of the intensity of the resting motor threshold for participants in this experiment, when the same TMS stimulator was applied over M1 outside the experiment). Previous studies have shown that, with these stimulation parameters, such bursts of TMS are able to induce transient disruptions of neural processing in FEF (Muggleton et al., 2003; O'Shea et al., 2004; for effects of the same TMS protocol in the current paradigm when applied over PPC instead of FEF, see Hung et al., 2005).

Our choice of TMS timing parameters was constrained by having to match these to the previous PPC TMS dataset (Hung et al., 2005) to allow a direct comparison of FEF versus PPC sites for otherwise equivalent experiments. Use of TMS burst that overlaps with the visual stimulus and extends beyond is also a common choice when seeking to maximize the likelihood of an observed effect on visual performance. Further variants on the present paradigm could manipulate the TMS timing in more detail [e.g., using single pulse at specific points or even bipulses, as used in O'Shea et al. (2004)] to pinpoint the critical processing times for specific top-down control processes. Here, our aim was more simple: to compare the impact of the present FEF site to the previous PPC site of Hung et al. 
(2005) when using an identical protocol except for the change in TMS site.

Data analysis. The analysis procedures for raw performance scores and for the derived formal TVA parameters were exactly the same in the new frontal (FEF) experiment as in our preceding parietal (PPC) study (Hung et al., 2005), where an overview of Bundesen's TVA approach was given in some detail, including all of the underlying formulae (for the original exposition, see Bundesen, 1990; for a more recent update, see Bundesen et al., 2005; for previous applications of the same TVA approach to braindamaged patients, see Duncan et al., 1999; Peers et al., 2005). We note that the recent neural extension of TVA (NTVA) (Bundesen et al., 2005, 2011), which we describe briefly below in the Results, does not change the way that our data need to be analyzed to extract the key Bundesen parameters. For further analyses directly comparing our new frontal effects to the parietal effects previously obtained (Hung et al., 2005), we were able to use a within-participant analysis, as the present eight participants had also undergone the PPC protocol [i.e., they were eight of the nine participants in Hung et al. (2005)].

\section{Results}

All participants completed the tasks without adverse effects. The application of TMS bursts over FEF did not elicit blinking responses, motor twitches, or sensory paresthesias, similar to the absence of any adverse effects for PPC TMS in Hung et al. (2005).

\section{Partial-report experimental task: impact of TMS on raw scores}

As illustrated in Figure 3, the general pattern of performance was consistent with the expectations of Bundesen's TVA (and related NTVA). Regardless of TMS conditions, performance on the target-alone displays was better than on two-item displays $\left(F_{(1,7)}=\right.$ $59.0, p<0.0005)$. This indicates some overall limit in processing capacity, as expected. Moreover, for two-item displays, an extra target was generally more disruptive than an additional nontarget $\left(F_{(1,7)}=54.7, p<0.0005\right)$. This indicates selectivity that prioritizes task-relevant over task-irrelevant stimuli, leading to an extra task-relevant item being more disruptive than an extra taskirrelevant one. It is this aspect of the results that corresponds to top-down control in TVA (and NTVA) terms.

Further analysis of raw performance scores was conducted separately for single- and two-item displays. For the single-item (target alone) displays, raw performance scores were subjected to a $3 \times 2$ repeated-measures ANOVA with factors of TMS condition (left FEF, right FEF, or none) and target side (left or right). No significant terms were found, indicating that TMS bursts over left or right FEF did not affect performance for a single target presented alone (Fig. 3, first and sixth bars in each of the histograms).

For two-item displays, raw scores were initially subjected to a four-way $3 \times 2 \times 2 \times 2$ repeated-measures ANOVA, with factors of TMS condition, target side, display configuration (same- or opposite-hemifield presentation; i.e., whether stimuli were in a column or in a row), and relevancy of the accompanying item (nontarget or target). Unlike the results for single-item displays (see above paragraph), this did reveal several impacts of TMS condition (such as an interaction between TMS and relevancy, $F_{(2,14)}=5.09, p=0.028$, as well as higher-order interactions). Thus, TMS affected the raw scores for two-item displays, unlike the results for single-item displays (see above). But, given that we had three levels of TMS (none, left FEF, or right FEF), any TMS effects and interactions are best understood when considering two particular TMS conditions at a time. Left or right FEF TMS can each be compared separately to no TMS to determine whether either TMS site had any impact whatsoever relative to no
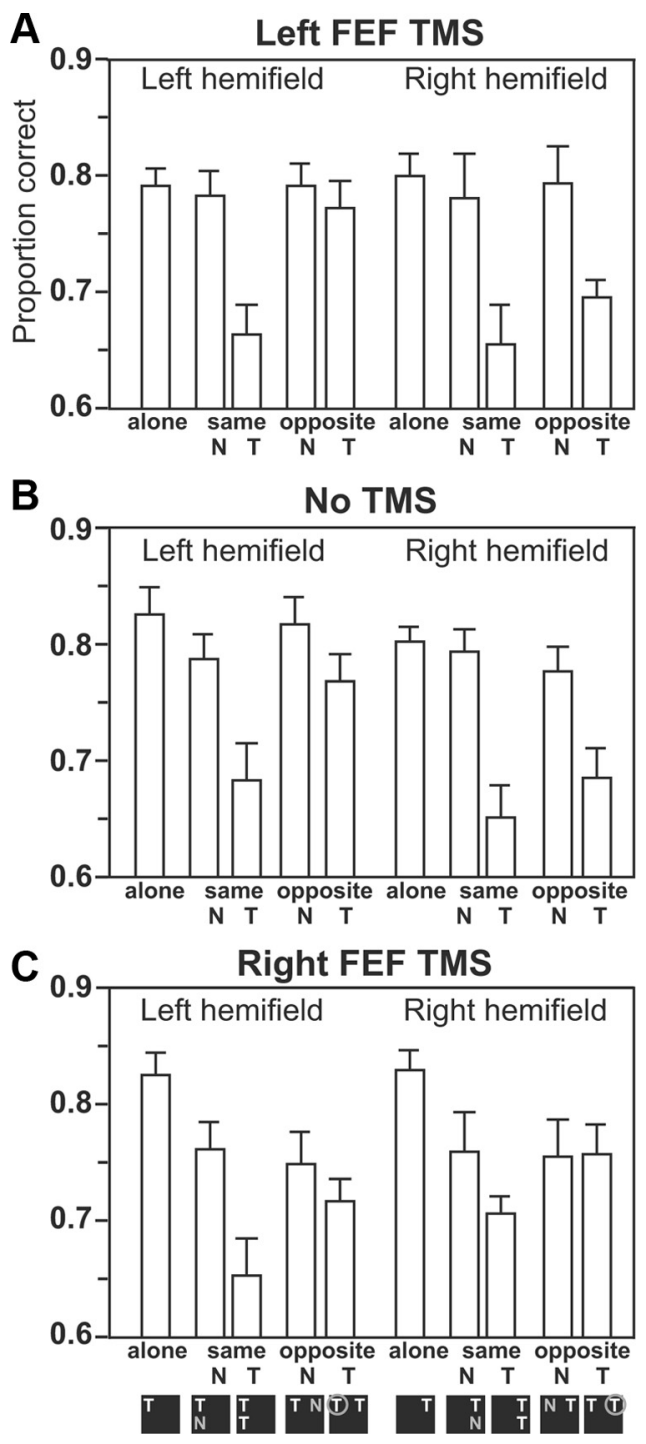

Figure 3. Mean values of raw performance scores in reporting the target (proportion correct) as a function of whether it appeared alone, with a nontarget (N) or target (T) in the same hemifield, or with a nontarget or target added in the opposite hemifield instead. Results shown for left FEF TMS $(\boldsymbol{A})$, no TMS $(\boldsymbol{B})$, or right FEF TMS $(\boldsymbol{C})$ in the visual selective partial-report task. Note the close similarity of left FEF TMS and no-TMS conditions, but the different pattern of results for right FEF TMS.

TMS. Moreover, comparing just left and right FEF TMS conditions directly should subtract out any potential nonspecific effects of TMS application per se, such as the click inevitably associated with TMS delivery (for an equivalent approach when comparing left PPC TMS, right PPC TMS, and none in a pairwise fashion, see Hung et al., 2005).

When comparing left FEF TMS to none, we found no impact on performance for raw scores from the two-item displays. A four-way ANOVA, now with just left FEF or none as the TMS factor (crossed with target side, row or column configuration, and relevancy of the accompanying item, i.e., nontarget or target) found only the usual main effects of relevancy and display configuration $\left(F_{(1,7)}=68.5, p<0.0005 ; F_{(1,7)}=10.7, p=0.01\right.$, respectively) with no significant terms involving the left-FEFversus-none TMS factor (all $F_{(1,7)}$ values $\leq 1.1$, all $p$ values $\geq 0.3$ ). Thus, left FEF TMS did not change the raw performance scores for the two-item displays, compared with no TMS (note the very similar patterns in Fig. 3, $A$ and $B$ ). 
Table 1. TVA parameters in the partial-report experimental task: values of sensory effectiveness, $A_{\mathrm{i}}$, spatial weights for targets $w_{\mathrm{i}}(T)$, and efficiency of control alpha for each individual participant

\begin{tabular}{|c|c|c|c|c|c|c|c|c|c|c|c|c|c|c|c|c|c|c|}
\hline \multirow[b]{3}{*}{ Participant } & \multicolumn{6}{|c|}{ Parameter $A_{\mathrm{i}}$} & \multicolumn{6}{|c|}{ Parameter $w_{\mathrm{i}}(T)$} & \multicolumn{6}{|c|}{ Parameter alpha } \\
\hline & \multicolumn{2}{|c|}{ Left FEF TMS } & \multicolumn{2}{|c|}{ No TMS } & \multicolumn{2}{|c|}{ Right FEF TMS } & \multicolumn{2}{|c|}{ Left FEF TMS } & \multicolumn{2}{|c|}{ No TMS } & \multicolumn{2}{|c|}{ Right FEF TMS } & \multicolumn{2}{|c|}{ Left FEF TMS } & \multicolumn{2}{|c|}{ No TMS } & \multicolumn{2}{|c|}{ Right FEF TMS } \\
\hline & Left & Right & Left & Right & Left & Right & Left & Right & Left & Right & Left & Right & Left & Right & Left & Right & Left & Right \\
\hline 2 & 2.12 & 1.48 & 2.22 & 1.67 & 2.48 & 1.69 & 0.87 & 0.68 & 0.90 & 0.79 & 0.78 & 0.74 & 0.58 & 0.22 & 0.53 & 0.29 & 0.88 & 0.80 \\
\hline 3 & 1.72 & 2.43 & 1.79 & 2.09 & 1.74 & 2.68 & 0.82 & 0.72 & 0.98 & 0.81 & 0.81 & 0.77 & 0.35 & 0.30 & 0.27 & 0.13 & 0.62 & 0.43 \\
\hline 4 & 2.49 & 2.45 & 2.86 & 2.36 & 2.56 & 2.34 & 0.91 & 0.73 & 0.92 & 0.79 & 0.88 & 0.90 & 0.42 & 0.22 & 0.39 & 0.22 & 0.58 & 0.24 \\
\hline 7 & 2.07 & 2.01 & 2.01 & 1.92 & 1.81 & 2.12 & 0.91 & 0.85 & 0.86 & 0.89 & 0.84 & 0.94 & 0.12 & 0.55 & 0.34 & 0.25 & 0.50 & 0.43 \\
\hline 8 & 2.09 & 2.22 & 2.60 & 2.16 & 2.38 & 2.42 & 0.85 & 0.60 & 0.83 & 0.85 & 0.59 & 0.95 & 0.34 & 0.73 & 0.44 & 0.23 & 0.92 & 0.48 \\
\hline
\end{tabular}

By contrast, when comparing right FEF TMS to no TMS in a similar four-way ANOVA, we found not only the usual main effects of relevancy $\left(F_{(1,7)}=48.7, p<0.0005\right)$ and configuration $\left(F_{(1,7)}=7.9, p<0.03\right)$, but also significant interactions involving the TMS factor of right FEF TMS versus none (interactions between TMS and target side: $F_{(1,7)}=15.6, p=0.006$; interactions between TMS and relevancy: $F_{(1,7)}=13.0, p=0.009$; three-way interaction between right FEF TMS vs none, with target side, and relevancy: $\left.F_{(1,7)}=12.1, p=0.01\right)$.

To identify the source of that three-way interaction, we collapsed over the uninvolved display-configuration factor, and then conducted separate 2 (right FEF TMS or none) $\times 2$ (target side) ANOVAs, either just for the conditions with an accompanying target, or just for those with an accompanying nontarget (which is what distinguishes the task-relevancy conditions). For conditions with an added nontarget, which is usually less disruptive to performance than an added target because nontargets do not have top-down relevance for the task, right FEF TMS actually worsened performance $\left(F_{(1,7)}=12.9, p=0.009\right)$. Right FEF TMS thus led to nontargets becoming more disruptive, indicating less top-down control, as confirmed more formally below. For conditions with an added target, a significant interaction $\left(F_{(1,7)}=\right.$ 23.7, $p=0.002$ ) between right FEF TMS (vs none) and target side revealed that right FEF TMS slightly enhanced performance in reporting the right target $(p<0.05)$ but led to a slight cost for the left target $(p<0.05)$, indicating some spatial influence of visual selection (Fig. 3).

Thus, right FEF TMS increased the detrimental impact of added nontargets overall, while also inducing a slight spatial advantage for right targets when presented together with left targets. This explains the three-way interaction of right FEF TMS $\times$ relevancy $\times$ target side.

The above analyses establish that, whereas performance did not differ between the left FEF TMS condition and no TMS, right FEF TMS changed the pattern from that for no TMS. Consistent with the equivalent pattern for no TMS and left FEF TMS, this impact of right FEF TMS is also revealed when comparing it directly to left FEF TMS instead. An ANOVA with 2 (right or left FEF TMS $) \times 2$ (target side $) \times 2$ (display configuration $) \times 2$ (relevancy of the accompanying item) structure found all of the same significant terms as when comparing right FEF TMS to none (see above), and for the same reasons, with significant interactions between right versus left FEF TMS and relevancy $\left(F_{(1,7)}=\right.$ $6.6, p=0.037)$; between side of TMS and target side $\left(F_{(1,7)}=10.1\right.$, $p=0.015)$; and the same three-way interaction between TMS site, relevancy, and target side $\left(F_{(1,7)}=10.7, p=0.01\right)$, as explained above.

\section{Analysis of formal TVA parameters derived from partial-} report experimental task

Parameters of TVA's best fits (for details of the approach and equations used for deriving these, see Bundesen, 1990; Duncan et al., 1999; Hung et al., 2005) were calculated for the different TMS conditions in each participant. The notation (Duncan et al., 1999; Hung et al., 2005) uses subscripts " $L$ " or "R" to indicate parameters for left or right hemifields, respectively; targets are labeled as " $\mathrm{T}$ " and nontargets as "N".

Parameters derived from upper and lower positions within the same hemifield were averaged together (Table 1). Best fits from TVA accounted for $72-95 \%$ (median $83 \%$ ) of the variance in the no TMS conditions, $70-89 \%$ (median $82 \%$ ) in the left FEF TMS conditions, and $72-85 \%$ (median $81 \%$ ) in the right FEF TMS conditions.

The Bundesen TVA parameter sensory effectiveness $\left(A_{\mathrm{i}}\right)$ was evaluated for left relative to right hemifields by the index $A_{\mathrm{L}} /\left(A_{\mathrm{L}}+\right.$ $\left.A_{\mathrm{R}}\right)$. Here, an index $>0.5$ implies a leftward bias in the overall effectiveness of visual processing, while $<0.5$ implies a rightward bias. See Figure $4 A$ for the distribution of this index in terms of the proportions of participants for a given score range (Duncan et al., 1999; Hung et al., 2005). No significant differences were found for this parameter among the three TMS conditions $\left(F_{(2,14)}=2.9\right.$, not significant). This indicates that TMS over the left or right FEF did not affect the basic sensory processing of a single target in either hemifield. Likewise, Hung et al. (2005) found that neither left nor right parietal TMS affected the Bundesen sensory-effectiveness parameter.

The next Bundesen TVA parameter to be examined was the overall spatial weighting of one hemifield versus the other, regardless of the distinction between targets and nontargets. This parameter and corresponding equations was calculated as described previously (Bundesen, 1990; Duncan et al., 1999; Hung et al., 2005). Intuitively, this parameter corresponds to whether one hemifield leads to better performance than the other, when disregarding the impact of the other parameters (such as the target/ nontarget distinction). This is evaluated by the index $w_{\mathrm{L}} /\left(w_{\mathrm{L}}+\right.$ $\left.w_{\mathrm{R}}\right)$, where $w_{\mathrm{L}}$ is averaged from $w_{\mathrm{L}}(N)$ and $w_{\mathrm{L}}(T)$, and $w_{\mathrm{R}}$ is derived similarly from $w_{\mathrm{R}}(N)$ and $w_{\mathrm{R}}(T)$. See Figure $4 B$ for the distribution of this parameter in terms of the proportion of subjects showing particular scores. There was no significant impact of TMS on this spatial-weighting parameter (computed regardless of target or nontarget status) among the three TMS conditions here $\left(F_{(2,14)}=2.9\right.$, not significant $)$.

The next and most critical Bundesen TVA parameter to be considered concerns the effectiveness of top-down control (corresponding intuitively to the differential impact of added 
A
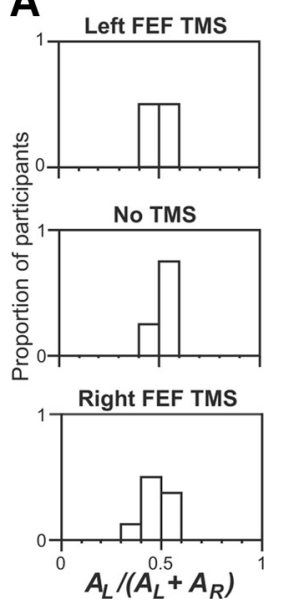

B

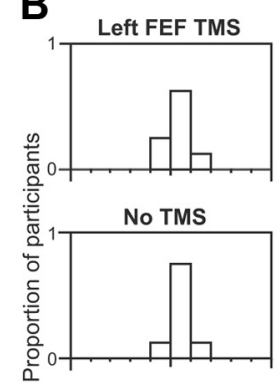

Right FEF TMS

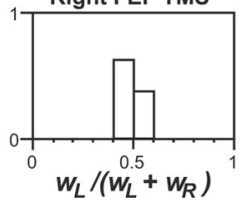

C

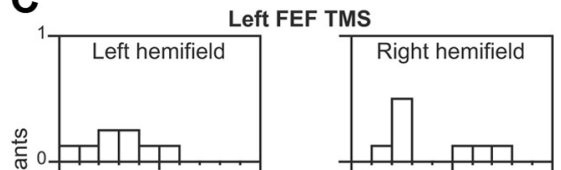

No TMS

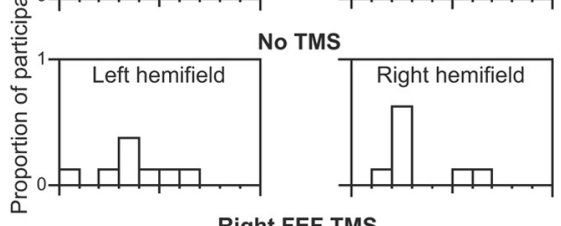

Right FEF TMS

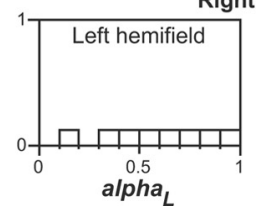

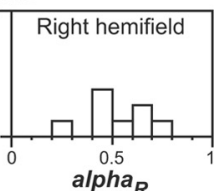

Figure 4. Distributions of various TVA parameters as proportions of participants (along $y$-axis) for each specified range of values (along $x$-axis). $\boldsymbol{A}$, The sensory effectiveness parameter, namely $A_{\mathrm{L}} /\left(A_{\mathrm{L}}+A_{\mathrm{R}}\right) . \boldsymbol{B}$, The spatial bias regardless of target/nontarget, namely $w_{\mathrm{L}} /\left(w_{\mathrm{L}}+w_{\mathrm{R}}\right)$. $\boldsymbol{C}$, The efficiency of top-down control for each hemifield, $\alpha_{\mathrm{L}}$ and $\alpha_{\mathrm{R}}$. The distribution for each parameter is shown separately for conditions of left FEF TMS (top), no TMS (middle), and right FEF TMS (bottom). The TMS conditions did not affect sensory effectiveness $(\boldsymbol{A})$ or spatial weighting $(\boldsymbol{B})$. However, for top-down control $(\boldsymbol{C})$ during right FEF TMS, the distribution of selectivity efficiency shifted for both hemifields (more subjects falling toward right of $x$-axis) for both $\alpha_{\mathrm{L}}$ and $\alpha_{\mathrm{R}}$, indicating impaired top-down control compared with left FEF or no TMS.
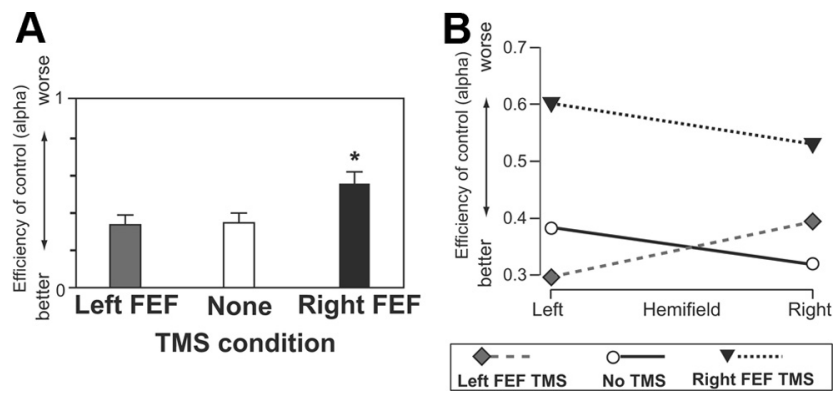

Figure 5. $\quad \boldsymbol{A}$, Mean values (with SE) of the TVA parameter $\alpha$ (efficiency of top-down control) for the three different TMS conditions, pooled over visual hemifield. Right FEF TMS led to significantly impaired top-down control (higher $\alpha$ score) compared with both left FEF TMS and no-TMS conditions, which did not differ. $\boldsymbol{B}$, Alpha scores shown separately for each hemifield in each TMS condition, shown only for completeness since the effect of right FEF TMS did not interact with hemifield, being present for both. ${ }^{*} p<0.05$.

targets compared with added nontargets), termed $\alpha$, and derived from $w(N) / w(T)$ (for exact details and equations, see Bundesen, 1990; Duncan et al., 1999; Hung et al., 2005). Note that low values for $\alpha$ indicate reduced impact of added nontargets relative to added targets (and thus efficient top-down control, here by tas relevance of the color for each item); whereas higher $\alpha$ values indicate worse top-down control (nontargets increasingly having as much detrimental impact as targets). Figure $4 C$ plots the distribution of $\alpha$ (as the proportion of participants for a specific score range) for the three TMS conditions. Note the tendency for more high scores (Fig. 4C, right column) to appear in the right FEF TMS condition in particular (Fig. 4C, bottom row). The $\alpha$ scores were subjected to a $3 \times 2$ repeated-measures ANOVA, with factors of TMS condition (left FEF, right FEF, or none) and hemifield. This produced a significant main effect for the TMS factor $\left(F_{(2,14)}=8.7, p<0.004\right)$, but no effect for hemifield $\left(F_{(1,7)}=0.025, p>0.8\right)$ or interaction $\left(F_{(2,14)}=1.5, p>\right.$ $0.25)$. Further contrasts collapsed across the ineffective hemifield factor revealed that right FEF TMS significantly increased parameter $\alpha$ (thus corresponding to significantly worse top-down con-

trol) both when compared with no TMS and when compared with left PPC TMS ( $p<0.05$ for either). By contrast, no TMS and left FEF TMS did not differ for $\alpha$ scores. Figure $5 A$ summarizes this straightforward outcome for the $\alpha$ scores now pooled over hemifields; Figure $5 B$ further confirms that the result did indeed apply for each hemifield. Thus, right FEF TMS impaired the efficiency of top-down control (by task-relevant color) overall for both hemifields, whereas left FEF TMS again had no significant impact.

This result for the critical Bundesen TVA top-down parameter, $\alpha$, is consistent with specific aspects of the raw performance scores presented earlier. In particular, it accords with the pattern of right FEF TMS producing worse performance specifically with an added nontargets, which normally has less detrimental impact on performance than an added target due to efficient top-down control, as clearly found here with no TMS or with left FEF TMS. The relatively minor spatial effect of right FEF TMS found in the raw performance scores (advantaging right targets while disadvantaging left targets) did not significantly influence $\alpha$ scores, as it arose in target-target situations, rather than when comparing the differential impact of added targets to that of added nontargets.

Subsequent to the Hung et al. (2005) PPC TMS study on which the FEF TMS study was based, Bundesen and colleagues (2005) have proposed an extension of TVA, termed NTVA, which seeks to place their psychological model in the context of neural phenomena. They propose, for instance, how the number of neurons firing (or their rate of firing) might relate to specific aspects of the TVA model. They also (like many other attention theorists) acknowledge a potential role for subcortical as well as cortical structures in a wider attentional network. Given the present FEF TMS intervention, we cannot say too much about subcortical involvement, but we acknowledge that the FEF represent just one key node of a wider network. Here, we were able to compare the impact of FEF TMS directly against TMS to a different node in the network (PPC), as described in Meta-analysis comparing FEF TMS effects to PPC TMS data of Hung et al. (2005), below. While NTVA links TVA to the wider neuroscience of attention, it does not change the way our data need to be analyzed (because NTVA relies on the same underlying equations as TVA), and does not alter how the TVA parameters should be interpreted psychologically. More recently, Bundesen and colleagues (2011) have further extended NTVA to discuss its possible relation to visual short term memory. The possible role of FEF in visual working memory is of considerable interest (Wallentin et al., 2008), but could not be addressed directly here, in part because of the relatively low target set sizes used (maximum of two per display) and the lack of any delay manipulation. Instead, our paradigm was optimized for extracting the other TVA parameters and for direct comparison with Hung et al. (2005).

\section{Color-report control task}

The color-report control task was completed for all participants to confirm that TMS did not directly affect color perception. Raw performance scores were subjected to a $3 \times 2$ repeated-measures 
Table 2. The TVA parameter, sensory effectiveness $A_{\mathrm{i}}$ (color) in the color-report control task

\begin{tabular}{|c|c|c|c|c|c|c|}
\hline \multirow[b]{2}{*}{ Participant } & \multicolumn{2}{|c|}{ Left FEF TMS } & \multicolumn{2}{|l|}{ No TMS } & \multicolumn{2}{|c|}{ Right FEF TMS } \\
\hline & $A_{\mathrm{L}}$ (color) & $A_{\mathrm{R}}$ (color) & $A_{\mathrm{L}}$ (color) & $A_{R}($ color $)$ & $A_{\mathrm{L}}$ (color) & $A_{R}$ (color) \\
\hline 1 & 3.47 & 2.08 & 3.47 & 4.16 & 4.16 & 3.47 \\
\hline 2 & 1.27 & 0.83 & 1.27 & 0.94 & 1.39 & 0.98 \\
\hline 3 & 2.08 & 2.37 & 2.21 & 3.06 & 2.77 & 2.37 \\
\hline 4 & 1.86 & 3.47 & 2.77 & 1.27 & 2.77 & 1.39 \\
\hline 5 & 3.47 & 2.77 & 3.47 & 2.77 & 3.47 & 2.37 \\
\hline 6 & 1.86 & 1.27 & 1.86 & 1.11 & 1.52 & 1.27 \\
\hline 7 & 2.37 & 1.39 & 1.76 & 1.27 & 1.52 & 1.67 \\
\hline 8 & 2.08 & 2.37 & 1.86 & 2.55 & 1.52 & 2.08 \\
\hline
\end{tabular}

ANOVA with factors of TMS (right FEF, left FEF, or none) and hemifield of the stimulus. Neither main effects nor the interaction approached significance, confirming that the results from the selective partial-report task (see Analysis of formal TVA parameters derived from partial-report experimental task, above) cannot be due to TMS-induced impairments of color vision per se, consistent with what one would expect given our use of FEF TMS sites. Likewise, when computing the TVA parameter of sensory effectiveness, $A_{\mathrm{i}}$, but now for color processing in particular (Table 2$)$, there was no impact of TMS condition $(p=0.9)$.

\section{Meta-analysis comparing FEF TMS effects to PPC TMS data of Hung et al. (2005)}

Except for our new use of FEF TMS sites, the present protocol was equivalent to our previous study (Hung et al., 2005), where we had applied TMS to parietal sites in either hemisphere instead. This now allows a direct comparison of any differential impacts of frontal (FEF) versus parietal (PPC) TMS within the same paradigm and for the same participants [the eight participants in this study also underwent the Hung et al. (2005) protocol]. The critical new result found here was that right FEF TMS diminished top-down control (the Bundesen $\alpha$ parameter) for both hemifields, while left FEF TMS had no such impact. Hung et al. (2005) somewhat analogously found that right PPC TMS could affect the top-down $\alpha$ parameter, while left PPC TMS had no effect. But importantly, right PPC TMS affected top-down control $(\alpha)$ in a different way from that newly observed here for right FEF TMS. Specifically, Hung et al. (2005) found that right PPC TMS impaired top-down control (i.e., increased $\alpha$ ) only for the contralateral left hemifield, tending instead to improve top-down control for the ipsilateral right hemifield (decreased $\alpha$ ), particularly for row displays analogous to those that can induce extinction in right parietal patients (for details, see Hung et al., 2005). The present effects on top-down control $(\alpha)$ for the right FEF TMS thus appear qualitatively different from those found by Hung et al. (2005) for the right PPC TMS (apart from the shared finding that left-hemisphere TMS had no effect in either study). To confirm this apparent qualitative difference for the right-hemisphere TMS effects more formally, we statistically compared the two studies directly for the critical Bundesen $\alpha$ parameter. This parameter corresponds to efficiency of top-down control and was affected by right FEF TMS here, and by right PPC TMS in Hung et al. (2005), but in apparently different ways.

We ran a 5 (no TMS, left PPC, right PPC, left FEF, right $\mathrm{FEF}) \times 2$ (hemifield) ANOVA on the TVA $\alpha$ parameters from the present FEF study and from the previous PPC study (Hung et al., 2005). This yielded a main effect for the factor of TMS condition $\left(F_{(4,28)}=4.188, p=0.029\right)$ and a significant interaction $\left(F_{(4,28)}=\right.$ 5.18, $p=0.012)$. The interaction between TMS and hemifield
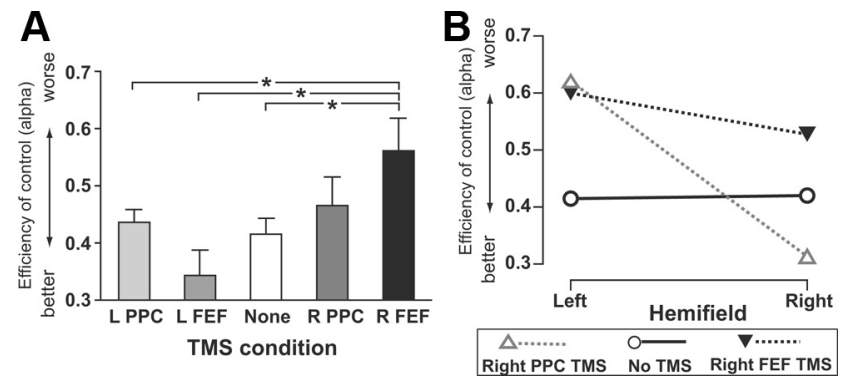

Figure 6. TMS effects top-down control ( $\alpha$ parameter) for left or right PPC TMS sites (taken from Hung et al., 2005) or for left or right FEF sites plotted together for comparison, using data from the same eight participants. The data for no TMS are pooled from the previous Hung et al. (2005) and the present experiment. $\boldsymbol{A}$, When pooling the data across hemifields, right FEF TMS differed from all other conditions except right PPC TMS. $\boldsymbol{B}$, The different outcome for top-down control under right PPC versus right FEF becomes apparent when plotting the $\alpha$ scores separately for left and right hemifield. While right FEF TMS (solid triangles) leads to high $\alpha$ scores (poor top-down control) in both hemifields, right PPC TMS (open triangles) leads to high $\alpha$ scores only for the contralateral left hemifield, producing instead unusually low $\alpha$ scores (enhanced top-down control) in the ipsilateral right hemifield. Thus, the impact of right FEF TMS on top-down control was equivalent for both hemifields, unlike the very different outcome for the two hemifields for right PPC TMS in Hung et al. (2005). * $p<0.05$.

reflects a significant difference between $\alpha$ values for left and right hemifields only for right PPC TMS (Hung et al., 2005). The main effect reflects worse top-down control overall during right FEF TMS, with right PPC as the next worse condition. Figure 6 plots the $\alpha$ data pooled over hemifield to illustrate the main effect of TMS.

As a further assessment of whether only right FEF TMS disrupted top-down control (leading to increased $\alpha$ parameters), regardless of hemifield [unlike the hemifield-dependent effect on $\alpha$ that Hung et al. (2005) found for right PPS TMS], each TMS condition from both papers was compared with the no-TMS baseline. No significant difference from no-TMS was found overall (when pooling $\alpha$ values across hemifields) for left PPC, right PPC, or left FEF TMS $\left(_{(7)}\right.$ ranging from 0.979 to 1.182 , associated $p$ values from 0.276 to 0.360$)$. The only significant overall decrease in top-down control (i.e., increased $\alpha$ ) across both hemifields was found for right FEF TMS versus none $\left(t_{(7)}=3.919, p=0.006\right)$, with a $35.5 \%$ mean increase in $\alpha$, corresponding to a decrease in the overall efficiency of top-down control. Thus, of all the four active TMS conditions, only right FEF TMS significantly disrupted the overall efficiency of top-down control when pooling across hemifields relative to no TMS.

\section{Discussion}

This study assessed the impact of TMS bursts (five pulses at 10 $\mathrm{Hz}$ ) over left or right human FEF on performance in a selective visual partial-report task, which required participants to identify target digits defined by the task-relevant color from a brief masked display. As used in psychophysical (Bundesen, 1990), neuropsychological (Duncan et al., 1999; Peers et al., 2005), or TMS (Hung et al., 2005) studies, this particular paradigm is well suited for characterizing distinct parameters underlying performance in a single task, via Bundesen's (1990) mathematical TVA [see also NTVA (Bundesen et al., 2005)]. Formal analysis in terms of TVA allows one to distinguish any impact of TMS on sensory effectiveness from potential impacts on spatial weighting of visual stimuli at different locations as distinct from potential impacts on top-down control (i.e., the additional attentional weight 
normally given to display items that are task-relevant rather than task-irrelevant, as distinguished here in an instruction-dependent manner by color).

Our new results showed that the FEF TMS had no impact on sensory effectiveness, nor on spatial-weighting aspects of visual performance, as derived by TVA. In contrast, top-down control was significantly disrupted, but only by right FEF TMS, with left TMS not differing from none. The impact of right FEF TMS on top-down control in particular was also apparent in statistically significant effects on the raw performance scores, before abstraction into TVA parameters. On one hand, the key pattern was that right FEF TMS led to added nontargets becoming more disruptive than normal. This indicates that nontargets are not filtered out as effectively as usual during right FEF TMS. This pattern was apparent for both hemifields, and indeed the impact of right FEF TMS upon $\alpha$ (the TVA parameter corresponding to top-down control) did not differ between hemifields. On the other hand, there was a spatial tendency for right FEF TMS to enhance report of ipsilateral right targets and to impair report of left targets in those displays that presented two targets in opposite hemifields [analogous to the extinction that has been reported to reveal spatial deficits after FEF damage in monkeys (Latto and Cowey, 1971)].

Our new finding that right FEF TMS disrupts top-down control significantly, and does so for both hemifields, adds to a growing literature indicating the importance of FEF, particularly within the right-hemisphere in humans, for modulation of visual processing (Grosbras and Paus, 2002, 2003; Muggleton et al., 2003; O'Shea et al., 2004; Silvanto et al., 2006; Taylor et al., 2007; Ruff et al., 2009). But our results go beyond previous work, adding new specificity in several ways as discussed below, notably with direct confirmation that it is indeed top-down control in particular that right FEF TMS disrupts when tested rigorously with the formal TVA model of Bundesen, which allows several different potential impacts upon visual processing to be separated mathematically, unlike more traditional performance measures.

\section{Effects from right- but not left-hemisphere FEF TMS in humans}

While right FEF TMS significantly disrupted top-down control, leading to nontarget stimuli becoming more disruptive, left FEF TMS has no such impact. This demonstrates that our results were specific to the particular TMS site, rather than reflecting a more trivial nonspecific impact (such as due merely to the clicking sound produced by any TMS application). In nonhuman primates, right and left FEF appear to be functionally analogous, with each primarily representing contralateral space more than ipsilateral space. However, the situation in humans may be different, as our study is by no means the first to report that right FEF TMS can have more marked influences on visual performance than left FEF TMS (Grosbras and Paus, 2002, 2003; Muggleton et al., 2003; O'Shea et al., 2004). Moreover, recent work combining TMS with concurrent fMRI has also reported that right FEF TMS in humans can have (bilateral) top-down effects on BOLD signals in visual cortex, which left FEF TMS does not produce (Silvanto et al., 2006; Ruff et al., 2009). Finally, it is well known that right-hemisphere brain damage in neurological patients (involving prefrontal, FEF, and/or parietal areas in a distributed network) can produce more severe visual dysfunctions than equivalent left-hemisphere damage; however, it has long remained unclear why this is so. Here, using TMS, we specifically showed, for FEF in particular, a causal role for human right but not left FEF in the top-down control function of weighting targets more highly than nontargets in terms of Bundesen's formal TVA. We also showed that the right FEF TMS effect on top-down control emerges for both visual hemifields.

\section{Differences between right FEF and right PPC TMS effects on top-down control of visual selection}

Because we used a protocol equivalent to Hung et al. (2005), changing only the site of TMS, we were able to compare our new FEF data directly against the previous PPC data to assess whether FEF TMS and PPC TMS have distinct effects. Hung et al. (2005) found that right (but not left) PPC TMS has a spatially specific impact on top-down control (the Bundesen $\alpha$ parameter), impairing top-down control in the contralateral left hemifield while paradoxically enhancing top-down control in the ipsilateral right hemifield, particularly for bilateral two-item displays. Thus, rather than impairing top-down control overall, right PPC TMS changed the spatial profile of top-down control. This contrasts with the present impact of right FEF TMS, which instead produced an overall disruption of top-down control (in particular, of filtering out nontargets) that was comparable in both hemifields. This reveals a qualitative difference between FEF and PPC influences and confirms Hung et al.'s (2005) speculation that, although right PPC may be involved in spatial modulation of top-down control, right frontal cortex (FEF in particular) might be critical for top-down control more generally, for both visual hemifields, as we found here.

A couple of patient studies have investigated TVA parameters in patients with frontal brain damage (Habekost and Bundesen, 2003; Peers et al., 2005). Unlike the present study, they were not able to target the FEF in particular. Perhaps due to the rather extensive lesions, top-down control parameters were not the only aspects of performance that differed in the patients. The present TMS approach allows a within-subject design that may be more sensitive to specific disruptions than the coarser between-groups comparisons often required in patient studies.

\section{FEF and distractor suppression}

The observed disruption of top-down control during right FEF TMS suggest insufficient rejection of nontargets in either hemifield, leading to worse performance in reporting any target with an accompanying nontarget. The integrity of right FEF function thus appears essential for suppressing distractors. Such a role for FEF may accord with evidence derived from single-unit recording studies in monkeys, in which Schall and colleagues (Schall and Hanes, 1993; Schall et al., 1995) found that activity of FEF neurons becomes increased or suppressed if their receptive fields contain a task-relevant target or task-irrelevant distractor(s), respectively, even in the absence of saccades. Within the population of FEF neurons, the location of a selected target can thus be signaled via the combined effect of increased activity for units whose receptive fields contain the target and suppression for those whose receptive fields contain distractors. It seems possible that TMS over the right human FEF disrupted some analogous distractor-suppression mechanism, although it must also be noted that in our human subjects, our effects were found only for right, not left, TMS (in accordance with many previous human TMS studies, see Effects from right- but not left-hemisphere FEF TMS in humans, above), and affected both hemifields during right FEF TMS. In further studies of FEF function in monkeys, Moore and colleagues (Moore and Fallah, 2001, 2004; Moore and Armstrong, 2003) have shown that FEF microstimulation can affect visual performance and exert top-down influences over 
responses in extrastriate visual cortex. Recently, Schafer and Moore (2011) further demonstrated in monkeys that voluntary control of FEF activity can alter spatial selection; while Zhou and Desimone (2011) documented a role for monkey FEF in nonspatial aspects of attention. Here, we used TMS in humans to show a specific influence on tightly defined top-down control of visual selection, as computed operationally by Bundesen's (1990) formal theory of visual attention.

In conclusion, our new data indicate that right human FEF is causally involved in top-down control of visual selection from brief displays in the absence of eye-movements, even when targets and nontargets are distinguished by a nonspatial property (here, color). FEF may thus be involved in both spatial and nonspatial aspects of visual selection (Zhou and Desimone, 2011). A direct comparison of our new results with Hung et al.'s (2005) findings for PPC TMS within the same paradigm established a qualitative difference between right FEF and right PPC influences. Right FEF TMS disrupted topdown control for both hemifields, unlike right PPC TMS, which disrupted top-down control contralaterally yet enhanced this ipsilaterally. Parietal cortex may be involved in spatial modulation of top-down control signals that originally emanate from the frontal eye fields, with the right-hemisphere being specialized for such top-down modulation of visual processing in humans.

\section{References}

Barone P, Batardiere A, Knoblauch K, Kennedy H (2000) Laminar distribution of neurons in extrastriate areas projecting to visual areas V1 and V4 correlates with the hierarchical rank and indicates the operation of a distance rule. J Neurosci 20:3263-3281.

Bichot NP, Schall JD (1999) Effects of similarity and history on neural mechanisms of visual selection. Nat Neurosci 2:549-554.

Bichot NP, Schall JD (2002) Priming in macaque frontal cortex during popout visual search: feature-based facilitation and location-based inhibition of return. J Neurosci 22:4675-4685.

Blanke O, Spinelli L, Thut G, Michel CM, Perrig S, Landis T, Seeck M (2000) Location of the human frontal eye field as defined by electrical cortical stimulation: anatomical, functional and electrophysiological characteristics. Neuroreport 11:1907-1913.

Bundesen C (1990) A theory of visual attention. Psychol Rev 97:523-547.

Bundesen C, Habekost T, Kyllingsbaek S (2005) A neural theory of visual attention: bridging cognition and neurophysiology. Psychol Rev 112:291-328.

Bundesen C, Habekost T, Kyllingsbæk S (2011) A neural theory of visual attention and short-term memory (NTVA). Neuropsychologia 49:1446-1457.

Campana G, Cowey A, Casco C, Oudsen I, Walsh V (2007) Left frontal eye field remembers "where" but not "what". Neuropsychologia 45:2340-2345.

Cornsweet TN (1962) The staircase method in psychophysics. Am J Psychol 75:485-491.

Duncan J, Bundesen C, Olson A, Humphreys G, Chavda S, Shibuya H (1999) Systematic analysis of deficits in visual attention. J Exp Psychol Gen 128:450-478.

Grosbras MH, Paus T (2002) Transcranial magnetic stimulation of the human frontal eye field: effects on visual perception and attention. J Cogn Neurosci 14:1109-1120.

Grosbras MH, Paus T (2003) Transcranial magnetic stimulation of the human frontal eye field facilitates visual awareness. Eur J Neurosci 18:3121-3126.

Habekost T, Bundesen C (2003) Patient assessment based on a theory of visual attention (TVA): subtle deficits after a right frontal-subcortical lesion. Neuropsychologia 41:1171-1188.

Hopfinger JB, Buonocore MH, Mangun GR (2000) The neural mechanisms of top-down attentional control. Nat Neurosci 3:284-291.

Hung J, Driver J, Walsh V (2005) Visual selection and posterior parietal cortex: effects of repetitive transcranial magnetic stimulation on partial report analyzed by Bundesen's theory of visual attention. J Neurosci 25:9602-9612.

Jenkinson M, Smith S (2001) A global optimisation method for robust affine registration of brain images. Med Image Anal 5:143-156.

Kawashima R, Tanji J, Okada K, Sugiura M, Sato K, Kinomura S, Inoue K, Ogawa A, Fukuda H (1998) Oculomotor sequence learning: a positron emission tomography study. Exp Brain Res 122:1-8.

Latto R, Cowey A (1971) Visual field defects after frontal eye-field lesions in monkeys. Brain Res 30:1-24.

Maunsell JH, Van Essen DC (1987) Topographic organization of the middle temporal visual area in the macaque monkey: representational biases and the relationship to callosal connections and myeloarchitectonic boundaries. J Comp Neurol 266:535-555.

Mesulam MM (1999) Spatial attention and neglect: parietal, frontal and cingulate contributions to the mental representation and attentional targeting of salient extrapersonal events. Philos Trans R Soc Lond B Biol Sci 354:1325-1346.

Moore T, Armstrong KM (2003) Selective gating of visual signals by microstimulation of frontal cortex. Nature 421:370-373.

Moore T, Fallah M (2001) Control of eye movements and spatial attention. Proc Natl Acad Sci U S A 98:1273-1276.

Moore T, Fallah M (2004) Microstimulation of the frontal eye field and its effects on covert spatial attention. J Neurophysiol 91:152-162.

Muggleton NG, Juan CH, Cowey A, Walsh V (2003) Human frontal eye fields and visual search. J Neurophysiol 89:3340-3343.

Murthy A, Thompson KG, Schall JD (2001) Dynamic dissociation of visual selection from saccade programming in frontal eye field. J Neurophysiol $86: 2634-2637$.

Oldfield RC (1971) The assessment and analysis of handedness: the Edinburgh inventory. Neuropsychologia 9:97-113.

O'Shea J, Muggleton NG, Cowey A, Walsh V (2004) Timing of target discrimination in human frontal eye fields. J Cogn Neurosci 16:1060-1067.

Paus T (1996) Location and function of the human frontal eye-field: a selective review. Neuropsychologia 34:475-483.

Peers PV, Ludwig CJ, Rorden C, Cusack R, Bonfiglioli C, Bundesen C, Driver J, Antoun N, Duncan J (2005) Attentional functions of parietal and frontal cortex. Cereb Cortex 15:1469-1484.

Rossi S, Hallett M, Rossini PM, Pascual-Leone A (2009) Safety, ethical considerations, and application guidelines for the use of transcranial magnetic stimulation in clinical practice and research. Clin Neurophysiol 120:2008-2039.

Ruff CC, Blankenburg F, Bjoertomt O, Bestmann S, Freeman E, Haynes JD, Rees G, Josephs O, Deichmann R, Driver J (2006) Concurrent TMSfMRI and psychophysics reveal frontal influences on human retinotopic visual cortex. Curr Biol 16:1479-1488.

Ruff CC, Blankenburg F, Bjoertomt O, Bestmann S, Weiskopf N, Driver J (2009) Hemispheric differences in frontal and parietal influences on human occipital cortex: direct confirmation with concurrent TMS-fMRI. J Cogn Neurosci 21:1146-1161.

Schafer RJ, Moore T (2011) Selective attention from voluntary control of neurons in prefrontal cortex. Science 332:1568-1571.

Schall JD, Bichot NP (1998) Neural correlates of visual and motor decision processes. Curr Opin Neurobiol 8:211-217.

Schall JD, Hanes DP (1993) Neural basis of saccade target selection in frontal eye field during visual search. Nature 366:467-469.

Schall JD, Thompson KG (1999) Neural selection and control of visually guided eye movements. Annu Rev Neurosci 22:241-259.

Schall JD, Hanes DP, Thompson KG, King DJ (1995) Saccade target selection in frontal eye field of macaque. I. Visual and premovement activation. J Neurosci 15:6905-6918.

Silvanto J, Lavie N, Walsh V (2006) Stimulation of the human frontal eye fields modulates sensitivity of extrastriate visual cortex. J Neurophysiol 96:941-945

Smith DT, Jackson SR, Rorden C (2005) Transcranial magnetic stimulation of the left human frontal eye fields eliminates the cost of invalid endogenous cues. Neuropsychologia 43:1288-1296.

Talairach J, Tournoux B (1988) Co-planar stereotaxic atlas of the human brain. Stuttgart: Thieme.

Taylor PC, Nobre AC, Rushworth MF (2007) FEF TMS affects visual cortical activity. Cereb Cortex 17:391-399. 
Tehovnik EJ, Sommer MA, Chou IH, Slocum WM, Schiller PH (2000) Eye fields in the frontal lobes of primates. Brain Res Brain Res Rev 32: 413-448.

Thompson KG, Schall JD (1999) The detection of visual signals by macaque frontal eye field during masking. Nat Neurosci 2:283-288.

Thompson KG, Hanes DP, Bichot NP, Schall JD (1996) Perceptual and motor processing stages identified in the activity of macaque frontal eye field neurons during visual search. J Neurophysiol 76:4040-4055.

Thompson KG, Bichot NP, Schall JD (1997) Dissociation of visual discrimination from saccade programming in macaque frontal eye field. J Neurophysiol 77:1046-1050.

Vuilleumier P, Driver J (2007) Modulation of visual processing by attention and emotion: windows on causal interactions between human brain regions. Philos Trans R Soc Lond B Biol Sci 362:837-855.

Wallentin M, Roepstorff A, Burgess N (2008) Frontal eye fields involved in shifting frame of reference within working memory for scenes. Neuropsychologia 46:399-408.

Wassermann EM (1998) Risk and safety of repetitive transcranial magnetic stimulation: report and suggested guidelines from the International Workshop on the Safety of Repetitive Transcranial Magnetic Stimulation, June 5-7, 1996. Electroencephalogr Clin Neurophysiol 108:1-16.

Zhou H, Desimone R (2011) Feature-based attention in the frontal eye field and area V4 during visual search. Neuron 70:1205-1217. 